Hydrol. Earth Syst. Sci., 17, 2487-2500, 2013

www.hydrol-earth-syst-sci.net/17/2487/2013/

doi:10.5194/hess-17-2487-2013

(C) Author(s) 2013. CC Attribution 3.0 License.

\title{
Circulation pattern based parameterization of a multiplicative random cascade for disaggregation of observed and projected daily rainfall time series
}

\author{
D. Lisniak ${ }^{1}$, J. Franke ${ }^{2}$, and C. Bernhofer ${ }^{2}$ \\ ${ }^{1}$ Federal Institute of Hydrology, Department of Water Balance, Forecasting and Predictions, Am Mainzer Tor 1, \\ 56068 Koblenz, Germany \\ ${ }^{2}$ Technische Universität Dresden, Faculty of Forest-, Geo- and Hydro Sciences, Institute of Hydrology and Meteorology, \\ Chair of Meteorology, Pienner Str. 23, 01737 Tharandt, Germany \\ Correspondence to: D. Lisniak (lisniak@bafg.de)
}

Received: 2 August 2012 - Published in Hydrol. Earth Syst. Sci. Discuss.: 5 September 2012

Revised: 3 May 2013 - Accepted: 24 May 2013 - Published: 5 July 2013

\begin{abstract}
The use of multiplicative random cascades (MRCs) for temporal rainfall disaggregation has been extensively studied in the past. MRCs are appealing for rainfall disaggregation due to their formal simplicity and the possibility to extract the model parameters directly from observed high resolution rainfall data. These parameters, however, represent the rainfall characteristics of the observation period. Since rainfall characteristics of different time slices are changing due to climate variability, we propose a parameterization approach for MRCs to adjust the parameters according to past (observed) or future (projected) time series. This is done on the basis of circulation patterns (CPs) by extracting a distinct MRC parameterization from high resolution rainfall data, as observed on days governed by each individual CP. The parameterization approach is tested by comparing the statistical properties of disaggregated rainfall time series of two time slices, 1969-1979 and 1989-1999, to the results obtained by two other disaggregation methods (a conceptually similar MRC without CP-based parameterization and a recombination approach) and to the statistical properties of observed hourly rainfall data. In this context, all three approaches use rainfall data of the time slice 1989-1999 for parameterization. We found that the inclusion of CPs into the parameterization of a MRC yields hourly time series that better reproduce the properties of observed rainfall in time slice 1989-1999, as compared to the simple MRC. Despite similar results of both MRCs in the validation period of 19691979, we can conclude that the CP-based parameterization
\end{abstract}

approach is applicable for temporal rainfall disaggregation in time slices distinct from the parameterization period. This approach accounts for changes in rainfall characteristics due to changes in the frequency of occurrence of the CPs and allows generating hourly rainfall from daily data, as often provided by a statistical downscaling of global climate change.

\section{Introduction}

A great variety of hydrological applications, including the modelling of urban water systems, require continuous rainfall series at hourly or even finer time steps as input. In this context two problems arise concerning the availability of input data at an appropriate temporal resolution: First, the available historical records are mostly present at a daily time step. The gauging network operated by the German Weather Service (DWD) for example includes roughly 5700 stations with rainfall records of daily values spanning back $100 \mathrm{yr}$. Compared to this, only $\sim 1200$ additional stations of the DWD network are capable of recording rainfall at hourly resolutions and an even smaller number has publicly available data covering more than $30 \mathrm{yr}$. Usually the hourly time series do not have an appropriate length for long-term simulations or contain gaps due to technical difficulties associated with high resolution rainfall measurement. The second problem of data availability arises when synthetic high resolution rainfall time series for projected climate scenarios 
are needed for climate change related impact studies. Possible local-scale realizations of precipitation time series for future climate scenarios are usually obtained by employing either dynamical or statistical downscaling methods on the output of general circulation models (GCM). However, only a few of these methods provide rainfall data at hourly resolutions, e.g. the regional climate models REMO (Jacob and Podzun, 1997) and CLM (Rockel et al., 2008). Most downscaling methods, including the widely adopted circulationpattern based approaches (e.g. Bárdossy et al., 2002; Enke et al., 2005; Yang et al., 2010), generate daily precipitation time series (e.g. Bárdossy et al., 2002; Enke et al., 2005; Yang et al., 2010) or are used to derive future possible changes in the spectrum of heavy precipitation for higher temporal resolutions (Franke and Bernhofer, 2009).

A common way to extend the data availability of hourly or sub-hourly rainfall at point locations is by stochastically generating synthetic rainfall time series with statistical properties similar to the observable high resolution rainfall at the location of interest. This is done either by generating synthetic rainfall time series "from scratch" or by performing a temporal disaggregation of available coarse scale rainfall amounts to finer time steps. The latter approach has a great practical relevance, since it makes use of the available daily information to generate possible realizations of diurnal rainfall patterns by redistributing the daily rainfall among finer time steps, so that the resulting high resolution rainfall values in turn add up to the given daily rainfall amount. Among the various methods for generating high resolution rainfall at point locations found in the literature, the most common are based on re-sampling procedures (e.g. Lall and Sharma, 1996; Vogel and Shallcross, 1996), point process theory (e.g. Glasbey et al., 1995; Koutsoyiannis and Onof, 2001) and scale invariance theory (e.g. Schertzer and Lovejoy, 1987; Olsson, 1998; Serinaldi, 2010).

Scale invariance theory, in particular, has received growing attention for temporal rainfall disaggregation purposes over the last $20 \mathrm{yr}$. Rainfall is assumed to be a multifractal process with scale invariant properties, i.e. the signal generated by the process shows statistical similarities across a limited range of scales (Olsson, 1995; Fraedrich and Larnder, 1993). For the theoretical basis of multifractality and scale invariance, we refer the reader to the works of Schertzer and Lovejoy (1987) and Lovejoy and Schertzer (2010a, b). The notion of multifractality and scale invariance for rainfall has been supported by a number of empirical studies (see e.g. Lovejoy and Mandelbrot, 1985; Hubert et al., 1993; Olsson et al., 1993; Tessier et al., 1996) and it is generally thought that this behaviour is inherited from the governing process of atmospheric turbulence. The first theoretical works aimed at supporting the empirical observation of multifractality in fully developed turbulence were done by Kolmogorov $(1941,1962)$ and multiplicative random cascades (MRCs) have since then been employed to model this highly intermittent multifractal process (Mandelbrot, 1974; Benzi et al., 1984; Frish, 1980). The interest in MRCs is related to their simplicity and because they themselves are generic multifractal processes (Lovejoy and Schertzer, 2010a). They have increasingly been used to model other multifractal processes, such as rainfall.

While MRCs have first mainly been used to model the spatial and temporal structure of rainfall (e.g. Lovejoy and Schertzer, 1990; Menabde et al., 1997), Olsson (1998) was the first to employ a discrete MRC with exact conservation of mass (i.e. microcanonical) for temporal rainfall disaggregation. The parameters of the proposed model, while being simplistic, were assumed to be dependent on two properties of the input time series, namely rainfall volume and position in the rainfall sequence. This assumption was made to better reproduce the autocorrelation of observed rainfall time series as well as to account for distinct internal structures of convective and frontal rainfall events, and was supported by the analysis of a two year rainfall record from southern Sweden.

Veneziano et al. (2006) deviated grossly from multifractality by introducing a cascade model with scale- and intensitydependent parameters, arguing that although the condensation of water vapour in the atmosphere inherits its multifractality from atmospheric turbulence, any scale invariance present in the condensation rate can be lost in the fall of condensed particles. These deviations from multifractality were consistently observed in rain gauge measurements and spacetime radar images and could be reproduced by explicitly accounting for scale- and intensity-dependency of the model parameters.

Rupp et al. (2009) extended this approach by modelling the functional dependency of model parameters on timescale and rainfall intensity by simple analytical functions, which resulted in a parameter-parsimonious cascade model. Serinaldi (2010) compared the results of a similar MRC to those obtained by a continuous universal multifractal model (CUM; see Schertzer and Lovejoy, 1987; Lovejoy and Schertzer, 2010a, b). While the MRC with its modifications could not be considered as multifractal at all, the results have shown a more accurate simulation of several properties of observed rainfall than could be achieved by the CUM model, which in fact is a more realistic resemblance of a multifractal process. This explains the further interest in the study and practical application of MRCs.

Despite the progresses made in rainfall modelling and disaggregation based on scale invariance theory, it has to be taken into account that the parameterization process for these models is data driven. Since the model parameters strictly represent the scaling behaviour of the climate region they were obtained from, with its predominant rainfall-generating mechanisms (Svensson et al., 1996; Güntner et al., 2001), it can be assumed that the same holds for the specific climate period. Given that the climate is dynamic and the predominant rainfall-generating mechanisms are subjected to variability according to a given climate change scenario, the aim of this paper is to propose a dynamic parameterization procedure for a discrete MRC based on atmospheric circulation 
patterns (CPs) and their frequency of occurrence. This parameterization approach is meant to enable the user to further disaggregate projected daily precipitation time series, which were generated by scaling down the synoptic scale output of general circulation models (GCMs) for a possible future climate, to hourly resolutions and to use them as input for climate impact studies.

This article starts with the description of the case study region and the sets of circulation pattern- and rainfall-data in Sect. 2. Section 3 introduces the models used in this study, while Sect. 4 describes the parameterization of the MRCs and their disaggregation procedure. In Sect. 5 the performances of the disaggregation models, especially CP-based MRCs, are assessed by comparison of several statistical properties of the observed and generated time series. In Sect. 6 we present our conclusion.

\section{Materials}

\subsection{Case study region and rainfall data}

Hourly rainfall data have been used for the parameterization and validation of the models. The data were obtained from a gauging station located approximately $400 \mathrm{~m}$ above sea level in the mountainous and forested catchment area of Wernersbach brook near Dresden, Germany, in the federal state of Saxony $\left(50.960873^{\circ} \mathrm{N}, 13.476405^{\circ} \mathrm{E}\right)$. With a mean annual precipitation of $853 \mathrm{~mm}$ (reference period 1961-1990), the local climate in Wernersbach brook is moderately wet, as compared to the region of Saxony with a mean annual precipitation of $600 \mathrm{~mm}$ in the reference period, which is due to the general increase of rainfall amounts with elevation (SMUL, 2008). The annual course of monthly precipitation shows the typical patterns of a temperate climate, with a primary peak in the summer season, where convective rainfall events are prevalent and a secondary maximum in the winter season, with rainfall events predominantly caused by frontal activity. A statistical analysis of climate trends for the study region has shown a decrease in precipitation of 10-20\% during the summer seasons of the period 1951-2000. The frequency of heavy precipitation events $\left(>20 \mathrm{~mm} \mathrm{~d}^{-1}\right)$ increased by a factor of 5 for the period 1971-2000, compared to 1961-1990 (Franke et al., 2004). This indicates shifting climatic conditions in the study region with effects on the probability distributions of hourly rainfall amounts in the first and second half of the period 1961-2000.

Hourly rainfall data at this location were available for two separate periods with (1) the period 1969-1979 covering 117 rainfall events of the summer season (April-September), and (2) the period 1989-1999 consisting of a complete rainfall time series of hourly rainfall amounts. The data of period (1) were recorded by a standard pluviograph with a $200 \mathrm{~cm}^{2}$ orifice and a $24 \mathrm{~h}$ recording strip. These rainfall records were manually digitized by first estimating the rainfall amounts ac- cumulated on each hour and dividing them by their total daily sum. Second, a time series of hourly rainfall for a given day was then obtained by weighting the rainfall amount accumulated over the day by a reference gauge with the hourly fractions, as calculated in the previous step. In period (2) rainfall amounts were digitized on site by a high-resolution tippingbucket instrument. This particular digitalization approach, however, can produce large differences in recorded rainfall intensity, since the rainfall depth recorded by a bucket tip is completely attributed to the time step where it occurred. Possible effects are a non-detection of periods with continuous rainfall characterised by low intensity or isolated time steps with overestimated rainfall intensity. Mascaro et al. (2013) provide a method to transform the discrete sampling signal of a tipping bucket instrument into a continuous representation of recorded rainfall. They analysed the intermittency and scaling behaviour of rainfall records obtained by both sampling strategies and found that rainfall intermittency is distorted by discrete sampling, while the scaling behaviour is not sensitive to a particular sampling strategy. We cannot account for a distorted rainfall intermittency by applying the transformation suggested by Mascaro et al. (2013), since records of the actual bucket tips are required for this. Unfortunately, such records are not available to us. Considering that the scaling behaviour remains unaffected by continuous or discrete sampling, methodical differences between these two digitalization techniques are assumed to be negligible at the temporal resolution of interest $(1 \mathrm{~h})$. Due to data availability the continuous rainfall record of period (2) was selected for model parameterization and thus termed the parameterization or calibration period. The rainfall events of period (1) were used to assess the performance of the models in reproducing the statistical properties of observed hourly rainfall amounts in a time slice distinct from the parameterization period and will be referred to as validation period throughout this paper. A continuous record of daily rainfall amounts was available from the reference gauge, covering the years 1969-1999 and has been used as input for the disaggregation process. Rainfall data of the winter season have been omitted from parameterization and the validation process due to the measurement errors, which have likely been introduced by snowfall occurrence, and the lack of winter rainfall data for the validation period.

\subsection{Circulation pattern data}

A time series of objective daily circulation pattern classes (CP), available for the period of 1961-2000, was used as a mean to couple the disaggregation process of the cascade model to a signal, which not only is available for the recent past climate but also can be derived from the outputs of GCMs for future climate scenarios. Those patterns were derived by an objective classification algorithm from the range of spatially averaged daily rainfall data and observed atmospheric predictor fields for the region of Saxony (Germany), 
Table 1. Class numbers of CPs and their intervals of spatially averaged daily precipitation according to Enke et al. (2005).

\begin{tabular}{cl}
\hline Class number & Class interval \\
\hline 1 & $0 \leq 0.05 \mathrm{~mm}$ \\
2 & $>0.05 \leq 0.5 \mathrm{~mm}$ \\
3 & $>0.5 \leq 1.0 \mathrm{~mm}$ \\
4 & $>1.0 \leq 3.0 \mathrm{~mm}$ \\
5 & $>3.0 \leq 5.0 \mathrm{~mm}$ \\
6 & $>5.0 \leq 10.0 \mathrm{~mm}$ \\
7 & $>10 \leq 15.0 \mathrm{~mm}$ \\
8 & $>15 \mathrm{~mm}$ \\
\hline
\end{tabular}

as described in detail by Enke et al. (2005), and thus are regionally valid for the study area. Briefly outlined, the classification method consists of dividing the spatially averaged time series of observed daily precipitation into eight moisture classes, with 1 being the "driest" and 8 the "wettest" class. According to Enke (2005), the intervals of the daily precipitation amounts for the $\mathrm{CP}$ classification are chosen as seen in Table 1 . In the next step, the eight empirically given classes are approximated by composite maps of meteorological predictor fields, as given by the ERA40 reanalysis (Uppala et al., 2005) for all days belonging to an individual moisture class. The predictor fields used for the approximation include the relative humidity at different levels and the vorticity at $850 \mathrm{hPa}$, which are related to the local precipitation amounts by bearing information about moisture content in the atmosphere and the formation of clouds (Enke et al., 2005). When using these composite maps of predictor fields to recognise CP classes from atmospheric fields, Enke et al. (2005) have shown that a majority of the CPs can be recognised correctly, with only $20 \%$ of the cases, most of them belonging to CP7 and CP8, being assigned to wrong classes. It can be hypothesised, this is due to the greater uncertainty in the composite maps of these CPs, stemming from a greater variability of precipitation events falling under wet conditions.

Thus the assumption was made that the locally recorded precipitation events can be coupled to synoptic scale properties of the atmosphere, which in turn yield information about the formation mechanisms of precipitation, i.e. convective or stratiform (Kronenberg et al., 2012). After the classification process and the construction of composite maps of predictor fields, these circulation pattern classes in turn can be recognised from the synoptic scale output of GCMs and from reanalysis data.

\section{Disaggregation models}

\subsection{Benchmark model}

A rainfall generator was used as benchmark to compare the disaggregation performance of the other models in the pa-

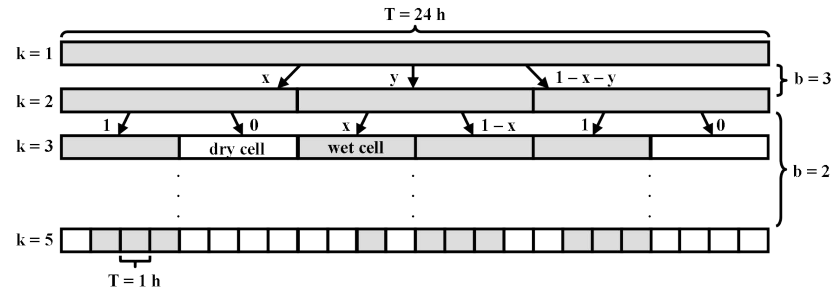

Fig. 1. Schematic of a cascade process, as implemented in the two cascade models presented in this paper. Cells of a cascade level $k$ with timescale $T_{k}$ are branched into a number of child cells, as defined by the branching number $b$, at the next cascade level $k+1$ and the rainfall volume of wet cells is redistributed among them according to rules defined by the cascade generator.

rameterization and validation period to a random recombination. The rainfall generator, which is explained in detail by Görner et al. (2009), extracts diurnal rainfall patterns of the parameterization period and bins them according to season and daily rainfall amount. The disaggregation of daily rainfall values is then performed by randomly choosing a diurnal rainfall pattern from an appropriate season and rainfall class and imprinting that pattern on the rainfall value to be disaggregated.

\subsection{Cascade models}

In this study, multiplicative random cascades (MRCs) are used for rainfall disaggregation. The models employed here are based on a discrete microcanonical MRC, as described by Olsson (1998) and Güntner et al. (2001). The implemented disaggregation process depicted in Fig. 1 basically consists of repetitively branching each discrete time interval (cell) of a rainfall time series at cascade level $k$ with a given timescale $T_{k}$ into a number of $b$ child cells of equal and subsequently smaller timescales $T_{k+1}=T_{k} b_{k}^{-1}$ at level $k+1$ and assigning each child cell a rainfall amount obtained by multiplying the rainfall volume $R_{k}$ of the parent cell by a weighting factor $W$. This redistribution of rainfall amounts is only performed for parent cells with rainfall volume $R>0 \mathrm{~mm}$ (wet cells), while the branching itself takes place for every cell of level $k$, including cells with $R=0 \mathrm{~mm}$ (dry cells). The number of child cells created can in principle assume any integer value $b \geq 2$, with $b=2$ being the most simple case. Since the structure emerging from this process resembles a tree, $b$ has been termed the branching number.

Starting with a series of daily rainfall values this procedure is successively repeated for each cascade level, until the desired timescale, in this case $T_{5}=1 \mathrm{~h}$, is achieved. It is obvious that this target timescale cannot be reached by subsequently doubling the starting temporal resolution of $T_{1}=24 \mathrm{~h}$, i.e. branching with constant $b=2$. The common cascade approaches found in the literature overcome this problem by defining a quasi-hourly target timescale (e.g. Güntner et al., 2001) or a quasi-daily starting timescale (e.g. Licznar 
et al., 2011). Since this was not an option in this work, the approach of a constant $b=2$ was abandoned in favour of a mixed branching with $b=3$ for the first cascade level $(k=1)$, resulting in a timescale of $T_{2}=8 \mathrm{~h}$ on the second cascade level. Branching of cascade levels $k>1$ then again is performed with $b=2$.

The generation of weights $W$ for the branching of wet cells is a random process, in which $W$ can assume a value in the closed interval $(0,1)$. However, the microcanonical feature of the MRC constrains the generation of weights in a way that the sum of $W \mathrm{~s}$ for child cells corresponding to a given parent cell is exactly 1 , i.e.

$\sum_{i=1}^{b} W_{i}=1$ with $b=\{2,3\}$.

Thus, conservation of mass between successive cascade levels is ensured. Furthermore, this means that in the case of $b=2$ only the weight $W_{1}$ of the first of the two child cells has to be determined. The weight of the second child cell, according to Eq. (1), is then $W_{2}=1-W_{1}$. In the case of $b=3$ however, the generation of weights becomes more complex, since the conservation of mass requires the range of possible values for $W_{2}$ to be conditioned on the value of $W_{1}$, with $W_{3}=1-W_{1}-W_{2}$.

The process of assigning random values for $W$ is termed the cascade generator. According to Olsson (1998), we defined several states that the cascade generator can assume with a given probability. Basically, these states bin together certain combinations of $W \mathrm{~s}$ that can be adopted by the child cells and they describe how the rainfall volume of a parent cell is redistributed among them. For $b=2$, three states could be defined

$W_{1}=\left\{\begin{array}{l}1 \text { with probability } P(1) \\ 0 \text { with probability } P(0) \\ x \text { with probability } P(x)\end{array}\right.$

where $x$ is associated with some probability distribution. Here, $W_{1}=1$ means that all the rainfall volume of the parent cell is assigned to the first child cell, while the second child cell remains dry (and vice versa for $W_{1}=0$ ). In the case of $W_{1}=x$ the rainfall volume of the parent cell is distributed between both child cells, where the fraction of the first cell equals $x$ and the fraction of the second cell $1-x$.

The number of states for the case $b=3$ has to be extended by incorporating the fraction of the second corresponding child cell on the aggregated rainfall volume to obtain sets of $W_{2}$ conditioned on values of $W_{1}$. Consequently, the weights $W_{1}, W_{2}$ and, for the sake of clarity, $W_{3}$ can assume seven states with their corresponding probabilities:
$W_{1}, W_{2}, W_{3}=$

$$
\left\{\begin{array}{ccccc}
1, & 0, & 0 & \text { with probability } & P(1,0,0) \\
0, & 1, & 0 & \text { with probability } & P(0,1,0) \\
0, & 0, & 1 & \text { with probability } & P(0,0,1) \\
x, 1-x, & 0 & \text { with probability } & P(x, 1-x, 0) \\
x, & 0, & 1-x & \text { with probability } & P(x, 0,1-x) \\
0, & x, & 1-x & \text { with probability } & P(0, x, 1-x) \\
x, & y, & 1-x-y & \text { with probability } & P(x, y, 1-x-y) .
\end{array}\right.
$$

The probabilities for the states of the cascade generator in Eqs. (2) and (3), as well as the probability distribution of $x$ and $y$, which will be both referred to as the parameters of the cascade model, can be derived directly from a time series of observed hourly rainfall amounts by inverting the cascade structure and aggregating the rainfall amounts through all cascade levels to daily rainfall sums. The weights $W$ are extracted in this process for each cascade level by dividing the rainfall volume in the first of the cells at level $k+1$ by the total aggregated volume $R>0 \mathrm{~mm}$ of a cell at level $k$. Following Olsson (1998), the probabilities $P(1), P(0)$ and $P(x)$ for every level $k$ are estimated by counting the occurrence of each state and dividing the number of occurrences by the total number of wet cells at the same cascade level. For $b=3$, this procedure is extended accordingly for the seven states in Eq. (3).

For all states where $x$ in Eqs. (2) and (3) is not 0 or 1, the empirical probability distributions of $x$ are estimated from the respective histograms of extracted cascade weights at every cascade level. The special case of $b=3$ and the state $\left(W_{1}\right.$, $\left.W_{2}, W_{3}\right)=(x, y, 1-x-y)$, however, requires the estimation of a probability distribution for $y$ conditioned on $x$. This is done by binning the known histograms of $x$ into 7 equidistant intervals and estimating the empirical probability distribution of $y$ for each of them, given that the corresponding $x$ is inside that interval.

The redistribution of rainfall amounts between the child cells can therefore be summarised as follows. For each wet cell in cascade levels 1 to 4 (1) Determine the properties (see Sect. 4) of the cell to be branched. (2) If the cell is a "wet cell", draw a uniformly distributed random number to determine the state of cascade generator for the branching of that particular cell according to the probabilities of states for the given cell properties. (3) Set the cascade weights according to the rules defined by the selected state. If the state of the cascade generator requires the weight to be set to $x$, determine this value using the empirical probability distribution of $x$ as defined for cells with the given properties. For $b=3$ and the state $\left(W_{1}, W_{2}, W_{3}\right)=(x, y, 1-x-y)$, first determine $x$ as described above, then determine $y$ using the probability distribution of $y$, as estimated for that particular range of $x$. (4) Distribute the rainfall volume of the parent cell to each 


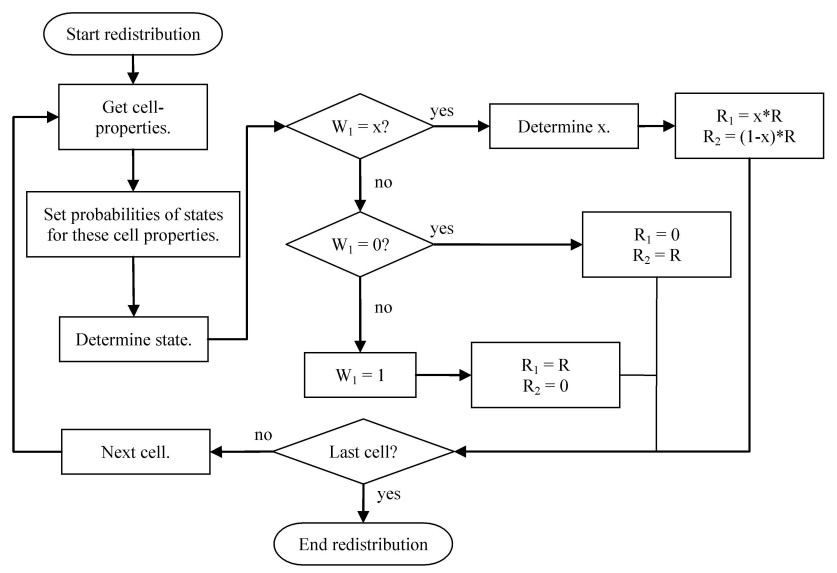

Fig. 2. Flowchart depicting the process of redistributing the rainfall volume of parent cells within a given cascade level between their child cells with branching number $b=2$.

child cell of the next cascade level by setting

$R_{i}=W_{i} R$ for $i=1 \ldots b$

with $R$ being the rainfall volume of a parent cell and $R_{i}$ of a child cell, where $i$ denotes the index of the $b$ child cells. This procedure is repeated for every cascade level, until the temporal resolution of $T_{5}=1 \mathrm{~h}$ is reached. See Fig. 2 for a depiction of this process for $b=2$.

\section{Parameterization of cascade models and disaggregation procedure}

\subsection{Parameter classification}

In order to improve the models ability to reproduce the internal structure of rainfall events, we assumed a dependency of the model parameters on parent cell properties (e.g. Olsson, 1998; Güntner et al., 2001; Rupp et al., 2009; Serinaldi, 2010). This is done by introducing a number of classes, each describing a certain characteristic of the cell to be branched, and by parameterizing one distinct cascade generator for every combination of the considered properties.

Since it has been shown by various authors that the variance of the cascade weights decreases with increasing temporal resolution (e.g. Marshak et al., 1994; Olsson, 1998; Paulson and Baxter, 2007), the first characteristic we considered is the timescale at level $k$. Hence the timescale property consists of four classes, corresponding to the temporal resolution of each cascade level.

To account for the distinct dependence of the probabilities $P$ of the different states and the probability distributions of $x$ on the rainfall volume of a parent cell, as well as the position of that cell inside the rainfall sequence (see Olsson, 1998; Güntner et al., 2001) a distinction between volume properties and position properties was employed. The four classes of the

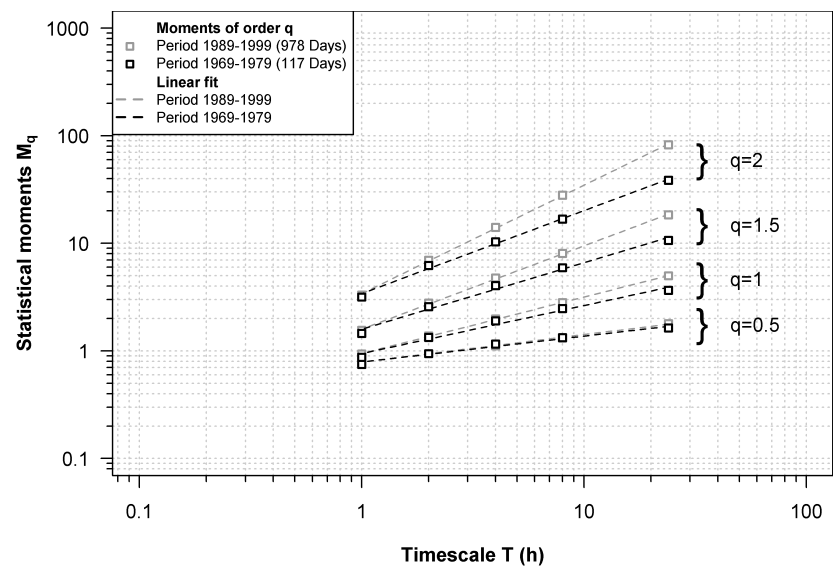

Fig. 3. Log-log plots of statistical moments $M_{q}$ of rainfall totals observed in the periods 1989-1999 (parameterization period) and 1969-1979 (validation period), respectively as a function of time $T$ between $T=1 \mathrm{~h}$ and $T=24 \mathrm{~h}$. Regression lines of the validation period are shifted and aligned with the intercepts of the parameterization period to avoid overlap of the plots. Actual log moments of both periods can bee seen in Table 2 .

position property are defined as follows (1) cells preceded by a dry cell and succeeded by a wet cell (starting class), (2) cells preceded and succeeded by wet cells (enclosed class), (3) cells preceded by a wet cell and succeeded by a dry cell (ending class), and (4) cells preceded and succeeded by dry cells (isolated class). The volume property consists of two classes of rainfall volumes (above mean and below mean). To distinguish between these two volume classes, the limit has been defined as the average rainfall volume of every sample of wet cells, which apart from the contained rainfall volume have similar remaining cell properties. The implications of this classification for the parameterization process are elaborated in Sect. 4.3.

\subsection{Coupling of generator parameters to a climate signal}

The parameter estimation process for cascade models found in the literature describes the scaling behaviour of observed rainfall, which not only is specific to the local climate, but also to the climate of the observation period. It is therefore assumed that the disaggregation of past or projected rainfall would imprint the scaling behaviour observed during the parameterization period on the target time slice, expecting that the scaling properties remain stationary between both periods. Since the climate system is in fact highly nonstationary, with short-term fluctuations and long-term trends (Rapp, 2000) and the resulting changes in the frequency and intensity of heavy precipitation events (Franke et al., 2004; Franke and Bernhofer, 2009), it can be argued that the parameters of a disaggregation model could be not transferable to different climatic periods. Considering the Wernersbach 
Table 2. Ordinary least squares estimation of scaling exponent $K(q)$ from statistical moments of rainfall in the parameterization period (1989-1999) and validation period (1969-1979) with the corresponding standard errors. Statistics of a t-test for differences in slope between the moment-scale relationships of the two periods are given below for 6 degrees of freedom.

\begin{tabular}{|c|c|c|c|c|c|}
\hline \multicolumn{6}{|c|}{ Parameterization period (1989-1999) } \\
\hline & \multirow{2}{*}{$\begin{array}{l}\text { Resolution } \\
\log (\mathrm{T})\end{array}$} & \multicolumn{4}{|c|}{ Moments of order $q$} \\
\hline & & $\log (\mathrm{M}(0.5))$ & $\log (\mathrm{M}(1))$ & $\log (\mathrm{M}(1.5))$ & $\log (\mathrm{M}(2))$ \\
\hline & 1.380211242 & 0.253028836 & 0.697977743 & 1.264183975 & 1.915700256 \\
\hline & 0.903089987 & 0.120219118 & 0.447806447 & 0.906196927 & 1.4471218 \\
\hline & 0.602059991 & 0.047682685 & 0.295841658 & 0.678548921 & 1.14775834 \\
\hline & 0.301029996 & -0.025656437 & 0.13914718 & 0.443370213 & 0.842998811 \\
\hline & 0 & -0.103238677 & -0.029567084 & 0.190521165 & 0.519347195 \\
\hline Slope $K(q)$ & & 0.256054507 & 0.524235805 & 0.774857527 & 1.008765484 \\
\hline \multirow{2}{*}{\multicolumn{2}{|c|}{ Standard error of slope }} & 0.004444608 & 0.004898782 & 0.01036128 & 0.010463118 \\
\hline & & \multicolumn{4}{|c|}{ Validation period (1969-1979) } \\
\hline & Resolution & \multicolumn{4}{|c|}{ Moments of order $q$} \\
\hline & $\log (\mathrm{T})$ & $\log (\mathrm{M}(0.5))$ & $\log (\mathrm{M}(1))$ & $\log (\mathrm{M}(1.5))$ & $\log (\mathrm{M}(2))$ \\
\hline & 1.380211242 & 0.491422508 & 1.089463531 & 1.774718298 & 2.542041268 \\
\hline & 0.903089987 & 0.40114778 & 0.920671511 & 1.520709617 & 2.182169703 \\
\hline & 0.602059991 & 0.341943452 & 0.805032797 & 1.354900179 & 1.97077079 \\
\hline & 0.301029996 & 0.25346042 & 0.651448879 & 1.158507545 & 1.749499254 \\
\hline & 0 & 0.153729687 & 0.467076792 & 0.909814024 & 1.456474399 \\
\hline Slope $K(q)$ & & 0.241889036 & 0.445364082 & 0.617350324 & 0.772296838 \\
\hline \multirow{3}{*}{\multicolumn{2}{|c|}{ Standard error of slope }} & 0.019459377 & 0.032770849 & 0.034804667 & 0.025925537 \\
\hline & & \multicolumn{4}{|l|}{ t-test statistics } \\
\hline & & $\mathrm{M}(0.5)$ & $\mathrm{M}(1)$ & $\mathrm{M}(1.5)$ & $\mathrm{M}(2)$ \\
\hline$t$ value & & 0.709674845 & 2.38031632 & 4.337344977 & 8.458209249 \\
\hline$p$ value & & 0.5045 & 0.0547 & 0.0049 & 0.0001 \\
\hline
\end{tabular}

rainfall data, differences in the scaling behaviour can be found for the two defined periods. This can be seen in Fig. 3, where the moments $M_{q}$ of order $q=\{0.5,1,1.5,2\}$ for the rainfall values of both periods are depicted as a function of time scale $T_{k}$. In a log-log plot the slopes of the moments of order $q$ become linear, which indicates a scale invariant behaviour in this range of scales. These slopes, which have been approximated using ordinary least squares regression (OLS), are estimates of the scaling function $K(q)$ for each individual moment of order $q$ (Lovejoy and Schertzer, 1995). The plots of the moments and the corresponding regression lines in Fig. 3 have been rearranged to avoid overlap and to highlight the differences of the slopes. The $K(q)$ values of the two periods are summarised in Fig. 4. Using a t-test, the significance of the differences has been confirmed for moments of order $q>1$ with a confidence greater than $95 \%$. The values used for the OLS, as well as the results of the regression and the t-test are listed in Table 1. Although Villarini et al. (2007) presented alternative regression frameworks to address the shortcomings of the OLS method and to better represent the uncertainty of $K(q)$, we think that since we con- sider only a limited number of moment orders and scales, the OLS method suffices for presentation purposes. Considering the differences of $K(q)$, it can be concluded that on average the daily rainfall values of the validation period scale down to larger hourly values, than those of the parameterization period. Additionally, the rainfall of the validation period is showing a higher variance. Consequently, it can be deduced from this analysis that the rainfall data of the validation period (1969-1979) is more influenced by heavy precipitation events than the parameterization period (1989-1999).

The proposed parameterization method attempts to account for this variability in the scaling properties of temporal rainfall in different time slices by coupling the model parameters to a climate signal. This climate signal consists of circulation patterns and specifically their frequencies of occurrence in different time slices. For the parameterization and validation period, these frequencies are shown in Fig. 5. The circulation patterns are appealing for this purpose, since classes of large-scale circulation patterns can be obtained from analysis of synoptic scale predictor fields of the recent climate re-analysis data (Enke et al., 2005; Yang et al., 


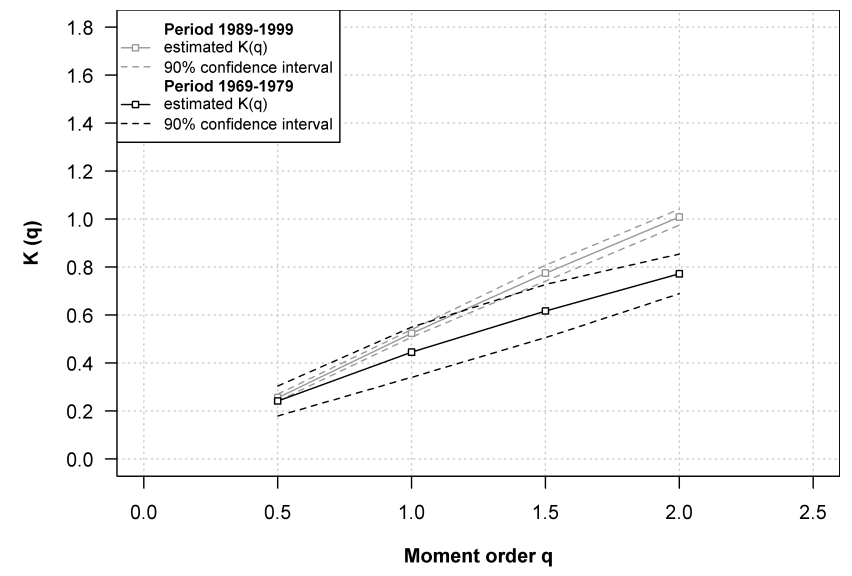

Fig. 4. Scaling exponent $K(q)$ as estimated from the moment-scale relationship of rainfall data in the periods 1989-1999 (parameterization period) and 1969-1979 (validation period) for moments of order $q=\{0.5,1,1.5,2\}$.

2010) and also, concerning the projection of model parameters into a possible climate future, from the output of GCMs, which have shown to provide a robust and consistent pattern of climate change (Mearns et al., 1999; Christensen, 2005). For this purpose, in addition to the parameter classification procedure of a simple cascade model as described in Sect. 4.1, a circulation-pattern-based approach has been implemented (CP-based cascade model). The CP-based cascade model introduces an additional cell property, the circulation pattern class predominant on a given day. This approach is based on the assumption that local heavy precipitation conditions are determined by atmospheric circulation patterns and their interaction with the local topography (Franke and Bernhofer, 2009) and therefore a difference in the circulation pattern frequencies in different time slices would lead to different scaling properties of temporal rainfall. Since the circulation patterns employed here are obtained from the ERA40 re-analysis using an objective algorithm, which in turn is optimised with regard to the precipitation regime, it can be hypothesised that the relationship between the circulation patterns and precipitation events is described properly inside the limits of the classification methods spatial resolution.

We justify this approach by the differences in temporal scaling of rainfall, as observed on days governed by each individual CP. Figure 6 shows the scaling functions $K(q)$ for CP 1 (the driest class) and CP 8 (the wettest class) of the parameterization period with their corresponding uncertainties. The scaling behaviour of the driest CP, having smaller $K(q)$ values, hints at greater hourly rainfall averages with a tendency for larger variances (Svensson et al., 1996), which is characteristic for convective events. The wettest $\mathrm{CP}$, on the other hand, shows scaling properties that favour longduration events with lower averages and variances of hourly rainfall compared to $\mathrm{CP} 1$. The wettest $\mathrm{CP}$ also exhibits a larger uncertainty, probably due to the smaller number of

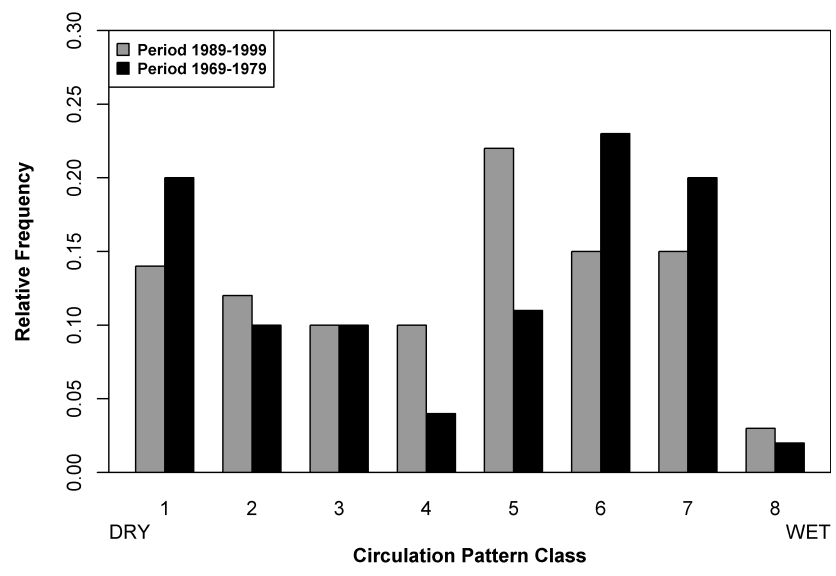

Fig. 5. Relative frequencies of circulation pattern classes for days with available hourly rainfall records in the periods 1989-1999 (parameterization period) and 1969-1979 (validation period).

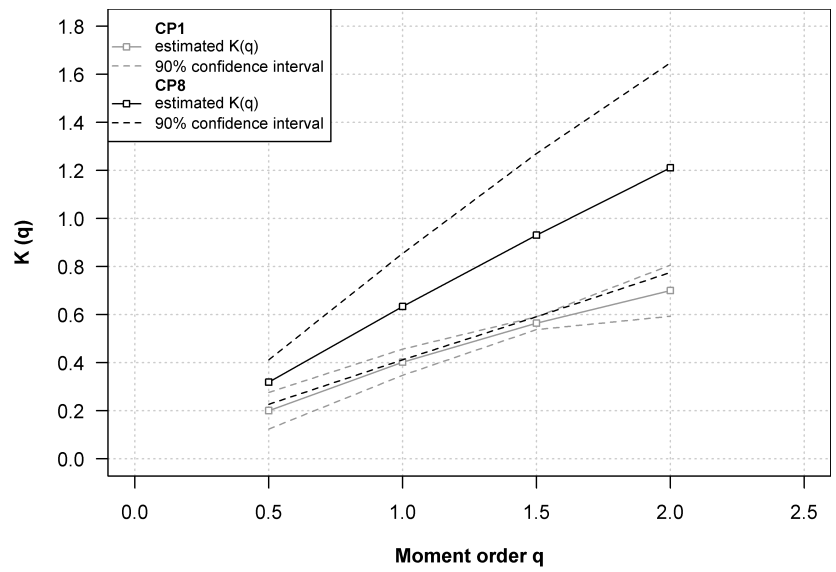

Fig. 6. Scaling exponent $K(q)$ as estimated from the moment-scale relationship of rainfall data on days governed by CP 1 and CP 8 in the period 1989-1999 for moments of order $q=\{0.5,1,1.5,2\}$.

rainfall events with greater variability covered by this CP. The other CPs (not shown) have their scaling properties in between these two extremes, while being less distinct.

\subsection{Parameterization}

In agreement with Enke et al. (2005), each wet cell has been classified according to the circulation pattern effective on that particular time step and binned into 8 classes. Following this, the cells in each CP class are classified with respect to the remaining three properties, i.e. in order of priority: position, timescale and volume. Ultimately the probabilities $P$ for the different states of the cascade generator as well as the probability distributions of $x$ and $y$ for each combination of cell properties were extracted. Since the number of parameters increased by factor 8 in the process, as compared to the parameterization without CPs, the average number of samples for each parameter decreased accordingly, which af- 
fects the uncertainty of the parameterization. The result of this procedure is a four-dimensional parameter matrix, as displayed in Fig. 7. Basically, this connotes the construction of distinct cascade generators for each $\mathrm{CP}$ (i.e. for each characteristic scaling regime) and thus weighting the parameters with the relative frequency of occurrence of circulation patterns. It is worth noting that while the frequency of the CPs may change between time slices, the relationship between MRC parameters and CPs is assumed to be invariant in time. A similar method has already been successfully employed for the derivation of a future temporal spectrum of heavy precipitation by Franke and Bernhofer (2009).

\section{Simulation results}

After parameterizing the MRCs and the rainfall generator on the time series of observed hourly rainfall for the period 1989-1999, 100 disaggregation runs were performed by each of the three models on daily rainfall data of time slices 1989-1999 (parameterization period) and 1969-1979 (validation period) to generate synthetic rainfall sequences at the hourly timescale. Additionally, daily rainfall amounts of the period 1979-1989, where no hourly rainfall records were available, have been disaggregated by both MRCs. The resulting sequences were analysed in terms of their ability to preserve several properties of the observed hourly rainfall time series in each period. The considered properties are (1) the exceedance probability $P(R>r)$ of hourly rainfall amounts, plotted as a cumulative density function (CDF), (2) the autocorrelation function (ACF) for lags one to ten and (3) the scaling of moments $M_{q}$ of order $q=\{0.5,1,1.5,2\}$. The CDFs for each model are constructed by computing the frequencies of occurrence of hourly rainfall amounts for every simulation run and building a composite by averaging the rainfall amounts for equal quantiles over the 100 realizations of the CDF. Equal quantiles were calculated using the Cunnane plotting position (Helsel and Hirsch, 1993). In the same manner, the 5th and 95th percentiles of the composite CDF for the synthetic time series were determined. For the ACF and the moment scaling behaviour, the average values of the 100 disaggregation runs have been calculated. The ACFs of the two cascade models are complemented with confidence intervals of the 5th and 95th percentiles from the ensembles. Here, the results of the simple rainfall generator function as a benchmark to assess the general applicability of the MRCs for rainfall disaggregation in time slices 19891999 and 1969-1979 in comparison to a more or less random recombination of diurnal rainfall patterns, as observed in the parameterization period. Furthermore, the results of both MRCs were compared to each other to evaluate the possible improvement of the synthetic time series generated by the CP-based cascade model in reproducing the properties of observed hourly rainfall in different time slices.

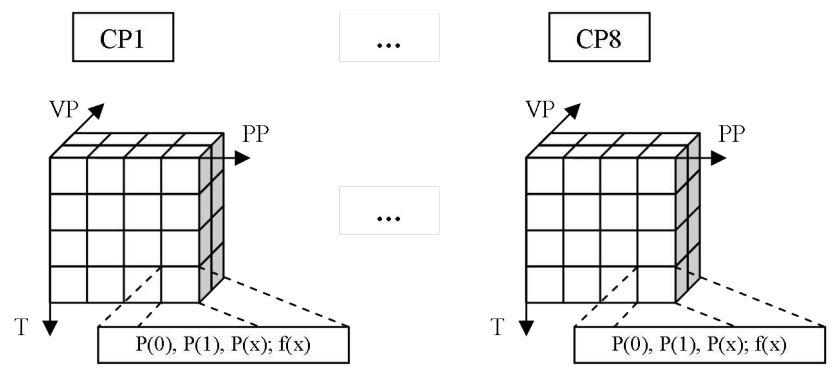

Fig. 7. Parameter set of a CP-based MRC for each combination of cell properties as a four-dimensional matrix. The small cubes contain the parameters for the branching of a parent cell with given cell properties CP (circulation pattern class), VP (volume property), PP (position property) and $\mathrm{T}$ (timescale property). A template of parameters for a branching with $b=2$ is shown for the highlighted cube. The parameters are the probabilities $P$ of states $W=0, W=1$ and $W=x$, and the empirical probability distribution function $f(x)$ for the state $W=x$.
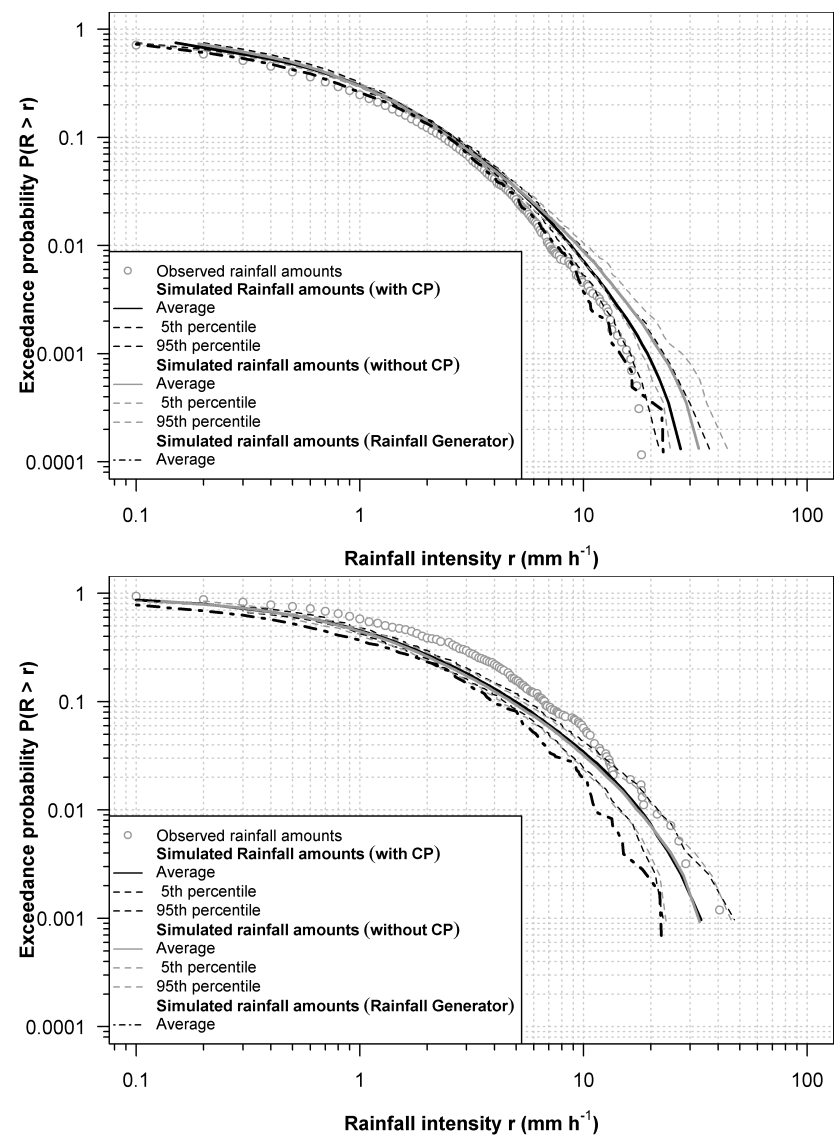

Fig. 8. Cumulative distribution functions of hourly rainfall amounts from observations and synthetic time series as generated by the cascade models (with and without CP) and the rainfall generator for parameterization period 1989-1999 (top) and validation period 19691979 (bottom). 
Concerning the ability of the simple rainfall generator to reproduce these properties it can be said, that the model performs exceptionally well for the parameterization period. The synthetic time series perfectly mimics the CDF of the observed hourly rainfall values (Fig. 8, top panel) as well as the moment scaling behaviour (Fig. 9, top panel) and, to a lesser degree, the ACF (Fig. 10, top panel). These results are expected, since the generated time series are a result of resampling observed diurnal rainfall patterns of the same time slice. Thus it is ensured that the properties of rainfall in this time slice are statistically preserved and bear the closest resemblance to observed data. This, however, is not the case for the validation period. Using observed diurnal patterns of time slice 1989-1999 to disaggregate daily rainfall values of the period 1969-1979 does not generate synthetic time series with properties close to those of the observed hourly rainfall. Figure 8 (bottom panel) shows that the CDF of the synthetic hourly rainfall amounts strongly underestimates the observed values by up to $50 \%$. Though the slopes $K(q)$ of the observed moments scaling behaviour in Fig. 9 (bottom panel) decreased, the moments of the synthetic time series appear to have similar slopes in both time slices, leading to an underestimation of the moments in the validation period. The ACF of the rainfall generator data for this time slice in Fig. 10 (bottom panel) overestimates the observed correlation for all lags, while showing behaviour that also is very similar to the parameterization period. This is due to the nature of the rainfall generator to imprint the diurnal rainfall patterns of the parameterization period on the target time slice. Therefore, the rainfall generator appears to be less suitable for disaggregation of daily rainfall values beyond the parameterization period.

The results obtained by both MRCs for the parameterization period do not reproduce the properties of observed hourly rainfall as well as the rainfall generator. Both cascade models slightly overestimate the CDF and underestimate the slopes $K(q)$ of the moment scaling behaviour of observed rainfall (Fig 8, top panel and Fig. 9, top panel), which is due to the tendency of cascade models to underestimate event durations (e.g. Olsson, 1998; Güntner et al., 2001; Rupp et al., 2009) and consequently producing hourly rainfall values with a higher variance than the observed data. This is also reflected by the slightly lower ACF values achieved by the cascade models (Fig. 10, top panel). However, considering that this disaggregation technique, in contrast to the rainfall generator, does not take any specific correlation structures into account, but indirectly preserves a degree of serial correlation due to the cascading structure (Molnar and Burlando, 2005), the ACF is being reasonably well reproduced by both cascade models. From the visual inspection of these plots it can be said, that both cascade models appear to be generally applicable for rainfall disaggregation in the parameterization period and that the introduction of a further source of complexity to the CP-based cascade model did not lead to a decline in performance while utilizing the statistical infor-
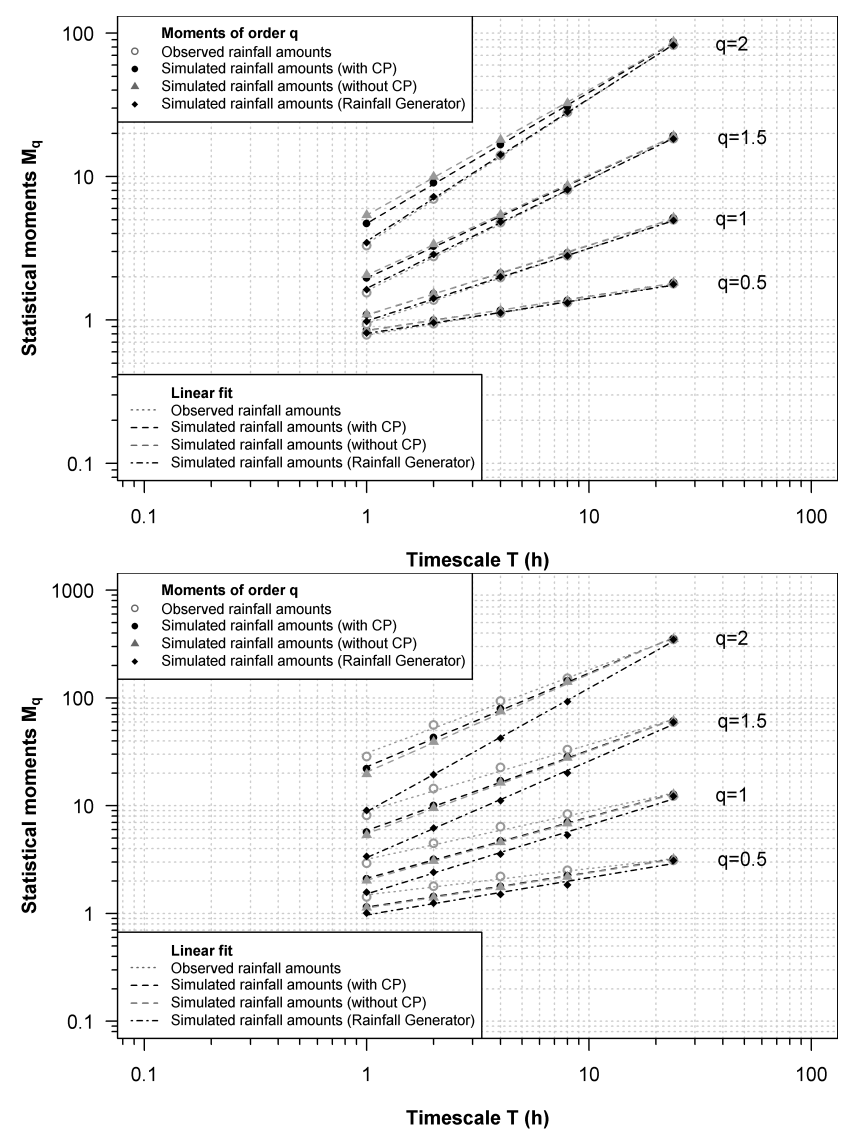

Fig. 9. Comparison of statistical moments $M_{q}$ of observed and synthetic rainfall data (averaged over 100 disaggregation runs) of the cascade models (with and without $\mathrm{CP}$ ) and the rainfall generator as a function of timescale $T$ between $T=1 \mathrm{~h}$ and $T=24 \mathrm{~h}$ for parameterization period 1989-1999 (top) and validation period 1969-1979 (bottom) in a log-log plot.

mation carried by the CPs. Although the differences between the ACFs and between the CDFs of both cascade models cannot be considered as statistically significant, the overestimation of rainfall appears to be less prominent for the CP-based MRC. It is worth noting that the results presented here for the disaggregation of Wernersbach daily rainfall values in the parameterization period could be reproduced for 14 different rain gauges inside and near Saxony (data not shown). Consistently, the output of the CP-based cascade model has shown a similar behaviour as described above, slightly better reproducing the properties of the observed hourly rainfall data. Due to sparse data availability at these rain gauges, only 5 years of hourly rainfall (1995-1999) could be used for parameterization, as well as disaggregation, so no test runs in different time slices could be made for these locations.

For the validation period, the results obtained by both MRCs are somewhat unexpected. While both cascade models generally perform better than the simple rainfall generator in terms of reproducing the frequency distribution of hourly 

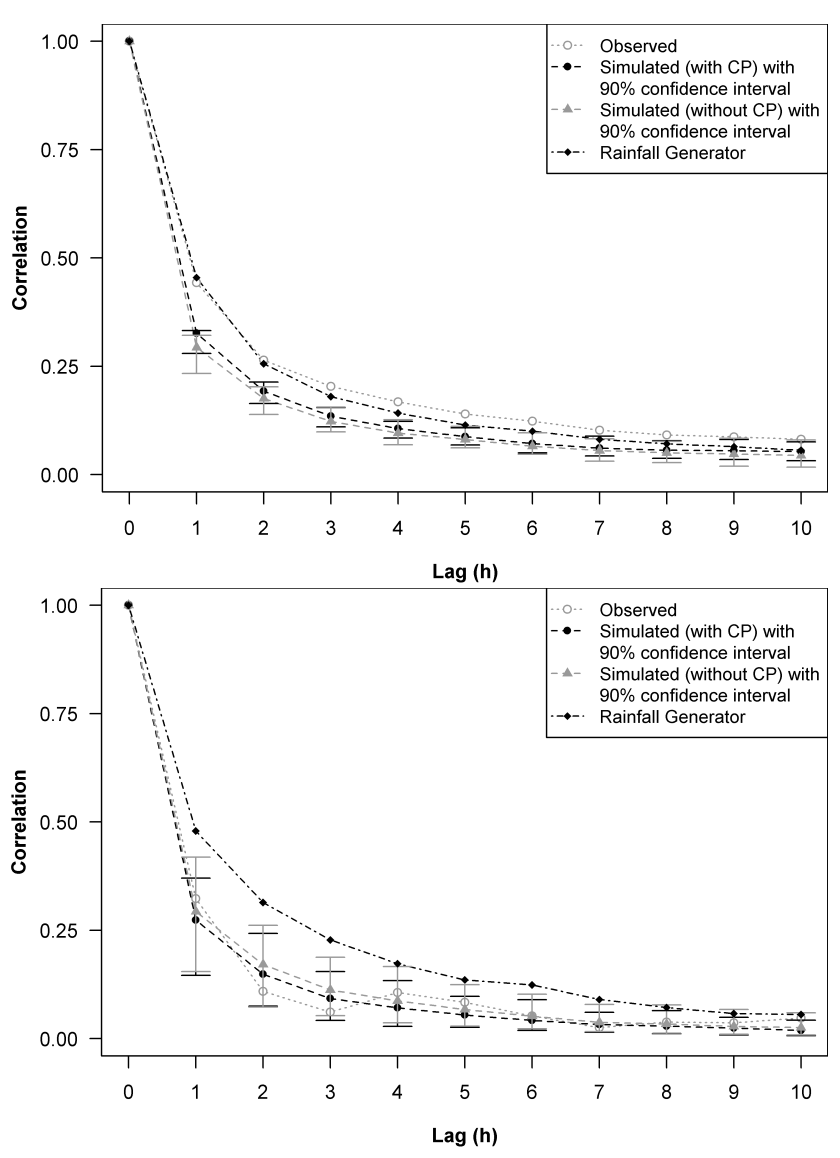

Fig. 10. Autocorrelation function of observed and simulated time series of hourly rainfall data (averaged over 100 disaggregation runs) of cascade models (with and without $\mathrm{CP}$ ) and the rainfall generator for parameterization period 1989-1999 (top) and validation period 1969-1979 (bottom). For the ensembles generated by the cascade models, $90 \%$ confidence intervals are given.

rainfall volumes (compare CDFs of cascade models, to the CDF of the rainfall generator in the bottom panel of Fig. 8), the moments scaling behaviour (Fig. 9, bottom panel) and the serial correlation (Fig. 10, bottom panel) of observed data, the properties of the time series generated by both cascade models are strikingly similar. In particular this is evident for the CDFs and the moment scaling behaviour of both synthetic time series in Fig. 8 (bottom panel) and Fig. 9 (bottom panel), suggesting that in this time slice both cascade models employ similar scaling mechanisms. To rule out the possibility that this behaviour is caused by the small sample size of the validation period, the disaggregation was repeated after extending the range of input data from 117 daily values, for which observed hourly rainfall data was available, to the whole range of 974 daily rainfall values available for the summer season of this period. The resulting CDFs of both cascade models (data not shown) here again displayed the same degree of similarity that can be observed in Fig. 8 (bottom panel) for the 117 disaggregated daily rainfall values.

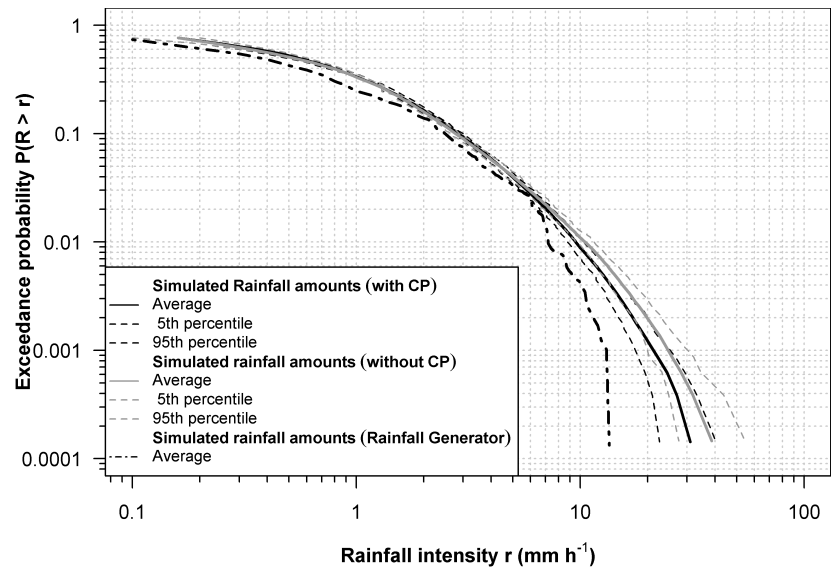

Fig. 11. Cumulative distribution functions of hourly rainfall amounts from synthetic time series as generated by the cascade models (with and without $\mathrm{CP}$ ) and the rainfall generator for the period 1979-1989. No observations of hourly rainfall amounts are available for this period.

Although we cannot rationalise adequately within the scope of this paper, why both MRCs produce similar results in the validation period, we can state that MRCs are better suited for rainfall disaggregation in time slices different from the parameterization period, than the simple rainfall generator. Despite the fact that no definite improvements in performance of the MRC could be observed in the validation period when accounting for synoptic scale conditions via a CP-based parameterization, it can be stated that the addition of another degree of freedom to the parameter set of the cascade model (in the form of atmospheric circulation patterns) apparently did not lead to a decrease in model performance below the benchmark of the simple cascade model. However, the synthetic time series generated by the CP-based MRC in the parameterization period, as well as the period 19791989 (Fig. 11), where no hourly rainfall values were available for validation, show a lower CDF than obtained by the simple cascade model. The results of the rainfall generator are added for comparison. Although no statements about the goodness of the synthetic time series in time slice 1979-1989 can be made, both MRCs here also yield distinct results. One possible explanation aims at the degree of spatial generalization involved in the objective classification method for circulation patterns that can be derived by direct comparison of point measurements of daily precipitation with the corresponding circulation pattern on that day and the interaction of local-scale-related vs. circulation-pattern-related effects on the local precipitation regime (Franke and Bernhofer, 2009). Since the circulation patterns employed in this paper are derived through an environment-to-circulation approach from spatially averaged precipitation amounts (Kreienkamp et al., 2010), some degree of uncertainty is implied in assigning a circulation pattern class for point locations which in fact has been shown to be less effective for the precipitation 
regime, due to the sub-scale processes involved that cannot be resolved by the underlying grid resolution of reanalysis data (Enke et al., 2005). Furthermore, the assignment of large-scale circulation patterns to one specific moisture class is a gross generalization, since weather conditions at point locations may be influenced by a composite of several circulation types and a fuzzy classification approach may be more appropriate for this matter. It can be hypothesised that these factors have led to a composite scaling behaviour of the CPbased MRC in time slice 1969-1979 that resembles the one obtained by the simple cascade model.

It should be noted, that the above results only account for statistics of the disaggregated rainfall data and not the random process of rainfall disaggregation itself. It has been shown by Lombardo et al. (2012) that the underlying random process of MRCs is characterised by intrinsic nonstationarity, since the autocorrelation function of the random process is not a function of lag only, as it would if the process was stationary. Hence they conclude MRCs do not preserve joint statistical properties of rainfall. As an alternative, they propose a downscaling approach based on a Hurst-Kolmogorov process and prove it to produce a stationary random process. While we do not account for the intrinsic non-stationarity of the MRCs used in this work, this problem should be mentioned and investigated in a future work.

\section{Conclusions}

We proposed a circulation pattern based parameterization procedure for a MRC, to be used for rainfall disaggregation from daily rainfall values to hourly resolution at point locations. This parameterization attempts to enable the cascade model to account for different rainfall generating mechanisms (i.e. rainfall caused by large-scale advection or local convection) and, with respect to variations in frequencies of these mechanisms due to climate change, for climate induced fluctuations in rainfall characteristics at high temporal resolutions. The results obtained by the MRC with CP-based parameterization were compared to a conceptually similar cascade model, whose parameters were not explicitly conditioned on circulation patterns and to a simple re-sampling approach.

We argue that, according to the findings of Olsson (1998), Rupp et al. (2009) and Serinaldi (2010), different combinations of wet cell properties generate a distinct scaling behaviour in cascade based rainfall disaggregation. Furthermore, we assume that the introduction of circulation patterns as an additional degree of freedom enables the MRC to account for climate induced variations in rainfall scaling behaviour, due to changes in the frequency of occurrence of synoptic scale circulation patterns. Since these circulation patterns can be derived from GCM outputs for future climate scenarios, the CP-based parameterization of MRCs, as derived from present or recent past rainfall data, can be dynam- ically projected to future time slices and used for the generation of possible realizations of future sub-daily rainfall time series. This amounts to weighting the model parameters by the frequency of occurrence of the circulation patterns, which has successfully been applied in the past to derive possible climate change related changes of statistical heavy precipitation (Franke and Bernhofer, 2009).

The method was tested by disaggregating daily rainfall amounts of the Wernersbach gauging station for the summer season of two time slices: The period 1989-1999 as calibration period (model parameterization), and 1969-1979 as the validation period. The results obtained with the CPbased cascade model have shown improvements in reproducing the autocorrelation function and the cumulative distribution function of observed hourly rainfall amounts for the time slice 1989-1999 as compared to the simple cascade model. Unexpectedly, for the validation period of time slice 19691979 the time series generated by both cascade models appeared to have similar properties. However, the performance of the CP-based cascade model did not drop beneath the benchmark of the simple cascade model, which is encouraging, since the introduction of an additional degree of freedom did not lead to a degradation of model performance in the validation period. Therefore, we conclude that the CP-based parameterization approach can be employed for disaggregation of rainfall in present and past climates, as well as projected time series of future daily rainfall values, which were obtained by a circulation pattern based downscaling method from GCM outputs.

Due to the sparse data availability, the proposed method could not be thoroughly validated for successive time series with observed hourly rainfall. Although the disaggregation of daily rainfall values with the CP-based MRC for a period without validation data (1979-1989) yielded time series with properties different from the ones obtained by the simple MRC, no statements can be made concerning the statistical goodness of the synthetic time series generated by both cascade models in that period. Therefore, it is essential to additionally test this parameterization approach for locations with several decades of available hourly rainfall data. Another important point for future work is to reanalyse the model results in view of the Hurst phenomenon (Koutsoyiannis, 2011) and its effects on rainfall disaggregation in different time slices. This, however, has to be backed up by a broader data basis.

Apart from this, further developments might deal with the CP-based parameterization of the cascade model as a fuzzy problem. This approach is appealing, since the weather conditions at point locations may be influenced by several classes of circulation patterns (Franke and Bernhofer, 2009). Additionally, the circulation pattern classes used in this work were obtained by an objective classification method, which may yield fuzzy assignments to individual circulation patterns for a given day (Enke et al., 2005). In this context, different regionally valid classification methods for circulation patterns should be tested with the CP-based 
parameterization approach to identify the most convenient classification method for describing the scaling regime of local precipitation.

Acknowledgements. The authors would like to thank Editor Bart van den Hurk, Francesco Serinaldi and an anonymous reviewer for their constructive comments, which significantly helped to improve the quality of the manuscript. Furthermore, the authors acknowledge Uwe Eichelmann and Heiko Prasse for maintaining the rainfall data and Klemens Barfuss for fruitful discussions. This work was funded by the Federal Ministry of Education and Research (BMBF) as part of the REGKLAM project (Grant ID.: 01 LR 0802) within the BMBF research focus KLIMZUG, supervised by the German Aerospace Center (DLR).

Edited by: B. van den Hurk

\section{References}

Bárdossy, A., Stehlík, J., and Caspary, H. J.: Automated objective classification of daily circulation patterns for precipitation and temperature downscaling based on optimized fuzzy rules, Clim. Res., 23, 11-22, 2002.

Benzi, R., Paladin, G., Parisi, G., and Vulpiani, A.: On the multifractal nature of fully developed turbulence and chaotic systems, J. Phys. A: Math. Gen., 17, 3521-3531, 1984.

Christensen, J. H.: Prediction of Regional Scenarios and Uncertainties for Defining European Climate Change Risks and Effects (PRUDENCE), Final Report, Contract No.: EVK2-CT2001-00132, DMI, Kopenhagen, 2005.

Enke, W., Schneider, F., and Deutschländer, T.: A novel scheme to derive optimized circulation pattern classifications for downscaling and forecast purposes, Theor. Appl. Climatol., 82, 51-63, doi:10.1007/s00704-004-0116-x, 2005.

Fraedrich, K. and Larnder, C.: Scaling regimes of rainfall time series, Tellus A, 45, 289-298, doi:10.1034/j.1600-0870.1993.t013-00004.x, 1993.

Franke, J. and Bernhofer, C.: A method for deriving a future temporal spectrum of heavy precipitation on the basis of weather patterns in low mountain ranges, Meteorol. Appl., 16, 513-522, doi:10.1002/met.149, 2009.

Franke, J., Goldberg, V., Eichelmann, U., Freydank, E., and Bernhofer, C.: Statistical analysis of regional climate trends in Saxony, Germany, Clim. Res., 27, 145-150, 2004.

Frish, U.: Fully developed turbulence and intermittency, Ann. N. Y. Acad. Sci., 357, 359-367, doi:10.1111/j.17496632.1980.tb29703.x, 1980.

Glasbey, C. A., Cooper, G., and McGechan, M. B.: Disaggregation of daily rainfall by conditional simulation from a point-process model, J. Hydrol., 165, 1-9, doi:10.1016/0022-1694(94)025986, 1995.

Görner, C., Franke, J., Bernhofer, C., and Goldberg, V.: Zwickauer Mulde River - Testing Regionalized Meteorological Forecasting/Scenarios, Report T21-09-02, Contract No.: GOCE-CT2004-505420, FLOODsite, Wallingford, 2009.

Güntner, A., Olsson, J., Calver, A., and Gannon, B.: Cascade-based disaggregation of continuous rainfall time series: the influence of climate, Hydrol. Earth Syst. Sci., 5, 145-164, doi:10.5194/hess5-145-2001, 2001.

Helsel, D. R. and Hirsch, R. M.: Statistical methods in water resources, Elsevier, Amsterdam, New York, 548 pp., 1993.

Hubert, P., Tessier, Y., Lovejoy, S., Schertzer, D., Schmitt, F., Ladoy, P., Carbonnel, J. P., Violette, S., and Desurosne, I.: Multifractals and extreme rainfall events, Geophys. Res. Lett., 20, 931-934, doi:10.1029/93GL01245, 1993.

Jacob, D. and Podzun, R.: Sensitivity studies with the regional climate model REMO, Meteorol. Atmos. Phys., 63, 119-129, doi:10.1007/BF01025368, 1997.

Kolmogorov, A. N.: The local structure of turbulence in an incompressible viscous fluid for very large reynolds numbers, Dokl. Akad. Nauk SSSR, 30, 301-305, 1941.

Kolmogorov, A. N.: A refinement of previous hypotheses concerning the local structure of turbulence in a viscous incompressible fluid at high Reynolds number, J. Fluid Mech., 13, 82-85, doi:10.1017/S0022112062000518, 1962.

Koutsoyiannis, D.: Hurst-Kolmogorov Dynamics and Uncertainty, J. Am. Water Resour. Assoc. , 47, 481-495, doi:10.1111/j.17521688.2011.00543.x, 2011.

Koutsoyiannis, D. and Onof, C.: Rainfall disaggregation using adjusting procedures on a Poisson cluster model, J. Hydrol., 246, 109-122, doi:10.1016/S0022-1694(01)00363-8, 2001.

Kreienkamp, F., Spekat, A., and Enke, W.: Ergebnisse eines regionalen Szenarienlaufs für Deutschland mit dem statistischen Simulationsmodell WETTREG201,. Final Report, Z4-BR - 50 201-2/4, Federal Environment Agency (UBA), Germany, 48 pp., 2010.

Kronenberg, R., Franke, J., and Bernhofer, C.: Classification of daily precipitation patterns on the basis of radar derived precipitation rates for Saxony, Germany, Meteorol. Z., 21, 475-486, doi:10.1127/0941-2948/2012/0343, 2012.

Lall, U. and Sharma, A.: A Nearest Neighbor Bootstrap For Resampling Hydrologic Time Series, Water Resour. Res., 32, 679-693, doi:10.1029/95WR02966, 1996.

Licznar, P., Łomotowski, J., and Rupp, D. E.: Random cascade driven rainfall disaggregation for urban hydrology: An evaluation of six models and a new generator, Atmos. Res., 99, 563578, doi:10.1016/j.atmosres.2010.12.014, 2011.

Lombardo, F., Volpi, E., and Koutsoyiannis, D.: Rainfall downscaling in time: Theoretical and empirical comparison between multifractal and Hurst-Kolmogorov discrete random cascades, Hydrol. Sci. J., 57, 1052-1066, 2012.

Lovejoy, S. and Mandelbrot, B. B.: Fractal properties of rain, and a fractal model, Tellus A, 37A, 209-232, doi:10.1111/j.16000870.1985.tb00423.x, 1985.

Lovejoy, S. and Schertzer, D.: Multifractals, Universality Classes and Satellite and Radar Measurements of Cloud and Rain Fields, J. Geophys. Res., 95, 2021-2034, doi:10.1029/JD095iD03p02021, 1990.

Lovejoy, S. and Schertzer, D.: Multifractals and rain, in: New uncertainty concepts in hydrology and water resources, edited by: Kundzewicz, Z., Cambridge University Press, Cambridge, United Kingdom, 62-103, 1995.

Lovejoy, S. and Schertzer, D.: On the simulation of continuous in scale universal multifractals, part I: Spatially continuous processes, Comput. Geosci., 36, 1393-1403, doi:10.1016/j.cageo.2010.04.010, 2010a. 
Lovejoy, S. and Schertzer, D.: On the simulation of continuous in scale universal multifractals, Part II: Space-time processes and finite size corrections, Comput. Geosci., 36, 1404-1413, doi:10.1016/j.cageo.2010.07.001, 2010b.

Mandelbrot, B.: Intermittent turbulence in self-similar cascades: divergence of high moments and dimension of the carrier, J. Fluid Mech., 62, 331-358, doi:10.1017/S0022112074000711, 1974.

Marshak, A., Davis, A., Cahalan, R., and Wiscombe, W.: Bounded cascade models as nonstationary multifractals, Phys. Rev. E, 49, 55-69, doi:10.1103/PhysRevE.49.55, 1994.

Mascaro, G., Deidda, R., and Hellies, M.: On the nature of rainfall intermittency as revealed by different metrics and sampling approaches, Hydrol. Earth Syst. Sci., 17, 355-369, doi:10.5194/hess-17-355-2013, 2013.

Mearns, L. O., Bogardi, I., Giorgi, F., Matyasovszky, I., and Palecki, M.: Comparison of climate change scenarios generated from regional climate model experiments and statistical downscaling, J. Geophys. Res., 104, 6603-6621, doi:10.1029/1998JD200042, 1999.

Menabde, M., Harris, D., Seed, A., Austin, G., and Stow, D.: Multiscaling properties of rainfall and bounded random cascades, Water Resour. Res., 33, 2823-2830, doi:10.1029/97WR02006, 1997.

Molnar, P. and Burlando, P.: Preservation of rainfall properties in stochastic disaggregation by a simple random cascade model, Atmos. Res., 77, 137-151, doi:10.1016/j.atmosres.2004.10.024, 2005.

Olsson, J.: Limits and characteristics of the multifractal behaviour of a high-resolution rainfall time series, Nonlin. Processes Geophys., 2, 23-29, doi:10.5194/npg-2-23-1995, 1995.

Olsson, J.: Evaluation of a scaling cascade model for temporal rainfall disaggregation, Hydrol. Earth Syst. Sci., 2, 19-30, doi:10.5194/hess-2-19-1998, 1998.

Olsson, J., Niemczynowicz, J., and Berndtsson, R.: Fractal Analysis of High-Resolution Rainfall Time Series, J. Geophys. Res., 98, 23265-23274, doi:10.1029/93JD02658, 1993.

Paulson, K. S. and Baxter, P. D.: Downscaling of rain gauge time series by multiplicative beta cascade, J. Geophys. Res., 112, D09105, doi:10.1029/2006JD007333, 2007.

Rapp, J.: Konzeption, Problematik und Ergebnisse klimatologischer Trendanalysen für Europa und Deutschland, phD thesis, Dt. Wetterdienst, Offenbach am Main, 2000.

Rockel, B., Will, A., and Hense, A.: The Regional Climate Model COSMO-CLM (CCLM), Meteorol. Z., 17, 347-348, doi:10.1127/0941-2948/2008/0309, 2008.

Rupp, D. E., Keim, R. F., Ossiander, M., Brugnach, M., and Selker, J. S.: Time scale and intensity dependency in multiplicative cascades for temporal rainfall disaggregation, Water Resour. Res., 45, W07409, doi:10.1029/2008WR007321, 2009.
Schertzer, D. and Lovejoy, S.: Physical Modelling and Analysis of Rain and Clouds by Anisotropic Scaling Multiplicative Processes, J. Geophys. Res., 92, 9693-9714, doi:10.1029/JD092iD08p09693, 1987.

Serinaldi, F.: Multifractality, imperfect scaling and hydrological properties of rainfall time series simulated by continuous universal multifractal and discrete random cascade models, Nonlin. Processes Geophys., 17, 697-714, doi:10.5194/npg-17-6972010, 2010.

SMUL (Ed.): Sachsen im Klimawandel - eine Analyse1, SMUL, Dresden, 209 pp., 2008.

Svensson, C., Olsson, J., and Berndtsson, R.: Multifractal Properties of Daily Rainfall in Two Different Climates, Water Resour. Res., 32, 2463-2472, doi:10.1029/96WR01099, 1996.

Tessier, Y., Lovejoy, S., Hubert, P., Schertzer, D., and Pecknold, S.: Multifractal analysis and modelling of rainfall and river flows and scaling, causal transfer functions, J. Geophys. Res., 101, 26427-26440, doi:10.1029/96JD01799, 1996.

Uppala, S. M., KÅllberg, P. W., Simmons, A. J., Andrae, U., Bechtold, V. D. C., Fiorino, M., Gibson, J. K., Haseler, J., Hernandez, A., Kelly, G. A., Li, X., Onogi, K., Saarinen, S., Sokka, N., Allan, R. P., Andersson, E., Arpe, K., Balmaseda, M. A., Beljaars, A. C. M., Berg, L. V. D., Bidlot, J., Bormann, N., Caires, S., Chevallier, F., Dethof, A., Dragosavac, M., Fisher, M., Fuentes, M., Hagemann, S., Hólm, E., Hoskins, B. J., Isaksen, L., Janssen, P. A. E. M., Jenne, R., Mcnally, A. P., Mahfouf, J. -., Morcrette, J. -., Rayner, N. A., Saunders, R. W., Simon, P., Sterl, A., Trenberth, K. E., Untch, A., Vasiljevic, D., Viterbo, P., and Woollen, J.: The ERA-40 re-analysis, Q. J. R. Meteorol. Soc., 131, 29613012, doi:10.1256/qj.04.176, 2005.

Veneziano, D., Furcolo, P., and Iacobellis, V.: Imperfect scaling of time and space-time rainfall, J. Hydrol., 322, 105-119, doi:10.1016/j.jhydrol.2005.02.044, 2006.

Villarini, G., Lang, J. B., Lombardo, F., Napolitano, F., Russo, F., and Krajewski, W. F.: Impact of different regression frameworks on the estimation of the scaling properties of radar rainfall, Atmos. Res., 86, 340-349, 2007.

Vogel, R. M. and Shallcross, A. L.: The moving blocks bootstrap versus parametric time series models, Water Resour. Res., 32, 1875-1882, doi:10.1029/96WR00928, 1996.

Yang, W., Bárdossy, A., and Caspary, H.: Downscaling daily precipitation time series using a combined circulation- and regression-based approach, Theor. Appl. Climatol., 102, 439454, doi:10.1007/s00704-010-0272-0, 2010. 